\title{
Thank you to reviewers 2017
}

The Editor would like to publicly acknowledge the people listed below who served as reviewers on the journal during 2016. Without their efforts, the quality of the journal could not be sustained.

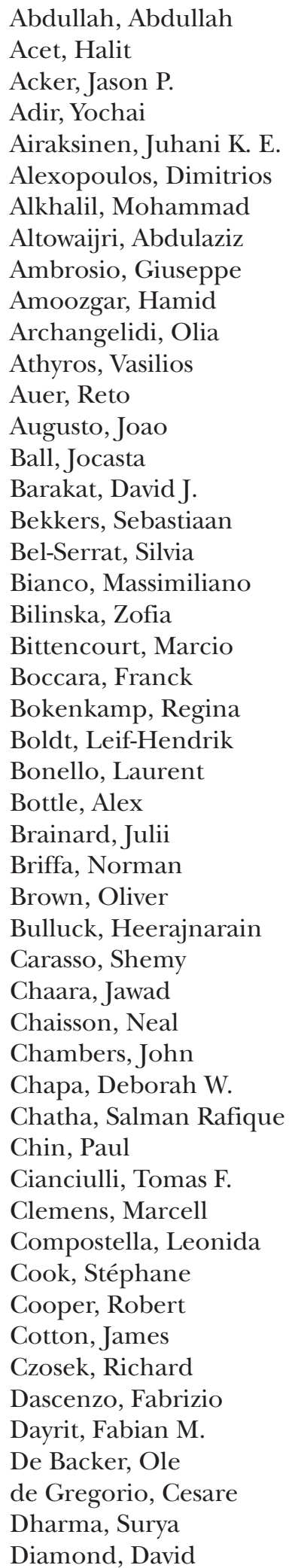

DiMauro, Michele

Donal, Erwan

Dong, Zhao

Dori, Guy

Droste, Dirk W.

Duval, Xavier

Egred, Mohaned

Elefteriades, John

Ellen, Dawson

Erne, Paul

Escobar-Diaz, Maria C.

Esposito, Giovanni

Feng, Quan-Zhou

Fernandez-Hidalgo, Nuria

Forsberg, Bertil

Francis, Heather M.

Garcia Fernandes, Miguel Angel

Garg, Pankaj

Gaynullina, Dina

Ghayour Mobarhan, Majid

Gierlotka, Marek

Gillies, Michael

Girardin, Francois

Gonzalez, Maday C.

Gorog, Diana

Guenoun, Maxime

Gunn, Jarmo

Guzik, Tomasz

Hambraeus, Krisina

Hamilos, Michalis

Hanzlikova, Hana

Harenberg, J

Harmon, Kimberly G.

Havranek, Thomas

Her, Sung-Ho

Heskethroberts, David

Hildick-Smith, David

Huang, Congxin

Huber, Christoph

Hulten, Edward

Indermuehle, andreas

Indermuehle, Andreas

Isilak, Zafer

Jans, Christian

Jalal, Zahraa S.

Jamieson, W. R. Eric

Jin, Hongfang

Johansen, Jens

Kaiser, Christoph
Kajander, Olli A.

Kang, Si-Hyuck

Karamfiloff, Kiril

Kasai, Takatoshi

Kashfi, F.

Keller, Frieder

Khera, Rohan

Kiefer, Todd L.

Kim, In Joo

Knapp, Guido

Ko, Dennis

Kobayashi, Shigeki

Kornej, Jelena

Korosoglou, Grigorios

Kovac, Jan

Kowalik, Ewa

Kreutz, Rolf P.

Kumar, Sathish

Kyhl, Kasper

Lambert, Laurie

Lang, Irene

Lanza, Gaetano

Lawrence, Glen

Leone, $\mathrm{S}$.

Leyva, Francisco

Lin, C. Huie

Linz, Dominik

Lip, Gregory

Lloris Carsi, Jose Miguel

Lowres, Nicole

Lundbye, Justin B.

MacIver, David

Magni, Paolo

Mair, Johannes

Malek, Lukasz A.

Malinski, Tadeusz

Marber, Michael

Marijon, Eloi

Mascherbauer, Julia

Mazurek, Michal

McCann, Gerry

McNeish, Alister J.

Menahem, Samuel

Metzler, Bernhard

Milani, Richard

Minamino, Tohru

Mockel, Martin

Moura, Marcia

Mutoh, Tatsushi

Narne, Parimala
Neyt, Mattias

Niccoli, Giampaolo

Nijjer, Sukhjinder S.

Nilsen, Dennis W. T.

Ntusi, Ntobeko

Okutucu, Sercan

Onat, Altan

Pagourelias, Efstathios

Pandit, Anil

Pandya, Bejal

Papadimitriou, Lampros

Parenica, Jirí

Parodi, Guido

Parthasarathy, Sairam

Patterson, C.

Pavy, B.

Perkins, Laura E. L.

Permanyer, Eduard

Philip, Femi

Pibarot, Philippe

Premaratne, Manuja

Providencia, Rui

Prutkin, Jordan

Puddu, Paolo Emilio

Pyxaras, Stylianos A.

Raja, Shahzad

Ramadi, Ailar

Rao, Sunil V.

Rasoul, S.

Ravnskov, Uffe

Reant, Patricia

Rechcinski, T.

Reddy, Gautam

Rey, Florian

Ribichini, Flavio

Rodriguez Palomares, Jose

Romero-Farina, G.

Sabaté, Manel

Sairaku, Akinori

Salgado, Angelo

Sandow, Shaun L.

Sasaki, Nobuo

Satoh, Toru

Schoenenberger, Andreas

Schulz, Richard

Schwab, Ursula

Seneff, Stephanie

Seo, Yoshihiro

Sezer, Murat

Singh, Thakur Uttam 
Sivakumaran, Krishna

Sobocinski, Piotr Doliwa

Sorkina, E. L.

Soulakova, Julia N.

Spiewak, Mateusz

Spratt, James

Sulo, Gerhard

Sultan-Taieb, Helene

Svennberg, Emma

Tarasov, R. S.

Tentolouris, N.
Teres, Cheryl

Timmers, Leo

Timoteo, Ana Teresa

Tobaldini, Eleonora

Toma, Mustafa

Tomizawa, Nobuo

Topilsky, Yan

Tossios, Paschalis

Vaislic, Claude

Vales, Lori

Valgimigli, Marco
VanGelder, Isabelle vanGeuns, Robert Jan Vanhaecht, Kris Voskuil, Michiel

Watanabe, Eiichi

Weaver, James C.

Weber, Alberto

Wenaweser, Peter

Westwood, Mark

White, Judith

Wilde, Arthur
Wilson, Lance D.

Wolny, Rafal

Xian, Ying

Yoshida, Kentaro

Yoshihisa, Akiomi

Zavalloni, Dennis

Zoller, Bengt

Zomahoun, Herve

Zongo, Arsene 